\title{
Mesoporous silica reinforced polybutadiene rubber hybrid composite
}

\author{
${\text { Madhuchhanda } \text { Maiti }^{1} \cdot \text { Ganesh C. Basak }^{1} \text { - Vivek K. Srivastava }}^{1}$. \\ Raksh Vir Jasra ${ }^{1}$
}

Received: 25 May 2015/Accepted: 7 October 2015/Published online: 13 April 2016

(c) The Author(s) 2016. This article is published with open access at Springerlink.com

\begin{abstract}
Polybutadiene rubber (BR) hybrid composites reinforced with mesoporous silica (MPS)/nanoclays, silica and MPS/carbon black were prepared. The primary focus of this research was to incorporate the mesoporous silicate (MPS), e.g., mobil composition of matter (MCM-41) as reinforcing filler in the BR matrix. The textural properties of the mesoporous materials were characterized by powder $\mathrm{X}$-ray diffraction (XRD), transmission electron microscopy and $\mathrm{N}_{2}$ isothermal adsorption measurements. The quantity of MCM-41 in the BR matrix was first optimized and the similar optimized quantity of different MPS was compared in terms of tensile strength. The composites were characterized by XRD and scanning electron microscopy. The composite containing $10 \mathrm{phr}$-loaded MCM-41 showed $250 \%$ improvement in tensile strength compared to the matrix devoid of nanomaterial. The effects of co-incorporation of two different kinds of nanomaterials having different nanostructures, e.g., layered montmorillonite and particulate MCM-41 were also studied. MCM-41 enhanced the mechanical strength of BR almost double the value compared to precipitated silica at the same filler loading. The morphological features of the composites were well corroborated with the mechanical properties.
\end{abstract}

Keywords Polybutadiene $\cdot$ MCM-41 $\cdot$ Hybrid composite $\cdot$ Mechanical properties

Madhuchhanda Maiti

madhuchhanda.maiti@ril.com

1 Reliance Technology Group, Vadodara Manufacturing Division, Reliance Industries Ltd., Vadodara, Gujarat 391346, India

\section{Introduction}

Elastomeric nanocomposites based on nano-sized inorganic particles and clusters have been paid more attention due to the interesting nano-sized effects of the particles [1]. Polymer nanocomposite shows unique properties, combining the advantages of the inorganic nanofillers (e.g., rigidity, thermal stability) and the organic polymers (e.g., flexibility, dielectric, ductility and processability) [2]. The nanoparticles will strengthen the matrix more than the conventional fillers due to high surface area-to-volume ratio and other fascinating properties. The mechanical and thermal properties of the composite will be significantly enhanced if there is a homogenous dispersion of the additives in the polymer matrix. This in turn, increases the interfacial adhesion between the polymer matrix and the nanofillers. Therefore, it is crucial and important to incorporate well dispersed nanofillers to the elastomer network to achieve superior physico- mechanical properties. At higher filler loading, the polymer-filler interaction decreases due to the agglomeration of fillers which ultimately reduces the strength of the composite. To increase the interaction between the matrix and the filler, a variety of methods can be applied such as functionalization or modification of the surface using some coupling agents [3].

Synthesis of mesoporous silica gained importance after the discovery of M41S type of molecular sieves by the scientists in the Mobil Oil Corp in 1990 [4]. These materials have perfectly long-range order, highly tunable pore size and good surface characteristics which make them an ideal material for various applications [5-7]. The polymer or reactive groups in the nano-sized pores extending along the channels to the openings will not only enhance the miscibility through the entanglement and inter-diffusion between the matrix and the particulate, but also highly 
suppress the aggregation of fillers. Though there are a few reports available in the literature regarding incorporation of the mesoporous silica (MPS) in different plastic matrix namely PP, PE, PANI, Epoxy, polycaprolactone, PMMA, PS [8-14]; the papers which describe the effect of MPSs in elastomers are very scarce $[5,15,16]$.

This paper discusses the effect of variable sized mesoporous silica as reinforcing filler in the polybutadiene rubber matrix. To the best of our knowledge, for the first time, we are reporting the effect of MPS on hybrid composites which contain other fillers like nanoclays, silica and carbon black. It is worth to investigate the synergistic effects of these fillers on the mechanical and dynamic mechanical properties of the hybrid composites.

\section{Experimental}

\section{Materials}

The mesoporous materials, mobil composition of matter No. 41 (MCM-41) and its precursor with template (MCM$\mathrm{T}$, without calcination), Santa Barbara Amorphous-15 (SBA-15) and mesocellular foam (MCF) were prepared inhouse as per the procedure reported in the literature [1719].

BR is supplied by Reliance Industries Limited, India and all the other chemicals are of analytical grade and they are used as such without any further purification. Pluronic P123, Cetyltrimethylammonium bromide (CTAB) and tetra methyl benzene (TMB) were purchased from Sigma-Aldrich, India. Sodium silicate, Zinc oxide, stearic acid, $N$-cyclohexyl-2-benzothiazolesulfenamide (CBS), bis- ( $\gamma$-triethoxysilylpropyl)-tetrasulfide (Si-69), precipitated silica, $N$-1,3-dimethyl-butyl- $n$-phenyl-paraphenylenediamines (6PPD), Micro crystalline wax, carbon black (N 330), diphenyl guanidine, naphthenic oil and sulfur were procured from Labort Fine Chemicals Limited, India. Nanoclay (Cloisite 20A, designated as MMT) was obtained from Southern Clay Products, USA.

\section{Preparation of composites}

The composites were prepared by mixing the following ingredients as per the recipe in Table 1 in a Brabender internal mixer followed by mixing the curatives in a two roll mixing mill for uniform mixing. All the compounds were vulcanized at $150{ }^{\circ} \mathrm{C}$ in a compression molding press at optimum cure time which was determined by a Monsanto Rheometer ODR 2000. All specimens were then accordingly cut from the vulcanized sheets.

\section{Characterization}

$X$-ray diffraction $(X R D)$

The mesoporous materials were characterized by X-ray Diffraction and Transmission electron microscopy. X-ray diffraction analysis were recorded with a Philips X'pert MPD system using $\mathrm{Cu} \mathrm{K} \alpha \mathrm{X}$-ray radiations $(\lambda=1.54056$ $\AA$ ) in $2 \theta=0.5^{\circ}$ to $10^{\circ}$ range in step size of 0.01 and a step time of $10 \mathrm{~s}$.

\section{Transmission electron microscopy (TEM)}

Transmission electron microscopy images of the mesoporous silica samples were captured with Titan 6300, FEI, USA, operating at a voltage of $300 \mathrm{kV}$. The samples were dispersed in acetone and then put on a carbon grid.

\section{Scanning electron microscopy (SEM)}

SEM samples were fractured in liquid nitrogen immersion and mounted with carbon tape wrapping. The images were studied with a Nova Nanosem 650, FEI, USA, instrument, operating at 1 and $10 \mathrm{kV}$ for the mesoporous silica and the composite samples, respectively.

\section{Surface area measurement}

The textual parameters such as surface area (BET), pore volume (PV), and pore diameter (dP) of calcined mesoporous silica samples were obtained from nitrogen adsorption data measured at $77.4 \mathrm{~K}$ using Micromeritics ASAP 2020 instrument. All the samples were degassed at $200{ }^{\circ} \mathrm{C}$ for $3 \mathrm{~h}$ prior to nitrogen adsorption. The specific surface area of the samples was calculated using the Brunauer-Emmett-Teller (BET) method in the relative pressure range $(\mathrm{p} / \mathrm{po})$ of $0.05-0.3$. The pore size distribution was determined using the Barrett-Joyner-Halenda (BJH) method, and pore sizes were obtained from the peak positions of the distributions curves.

\section{Mechanical properties}

Room temperature tensile tests were done by an Instron 3367 testing machine at a cross head speed of $500 \mathrm{~mm} / \mathrm{min}$ as per ASTM 412-97. The measurement was repeated for 3 times and the average value was reported.

\section{Hardness}

Hardness was determined on a $6 \mathrm{~mm}$ thick specimen on Durometer (Wallace) as per ASTM D2210-97. 


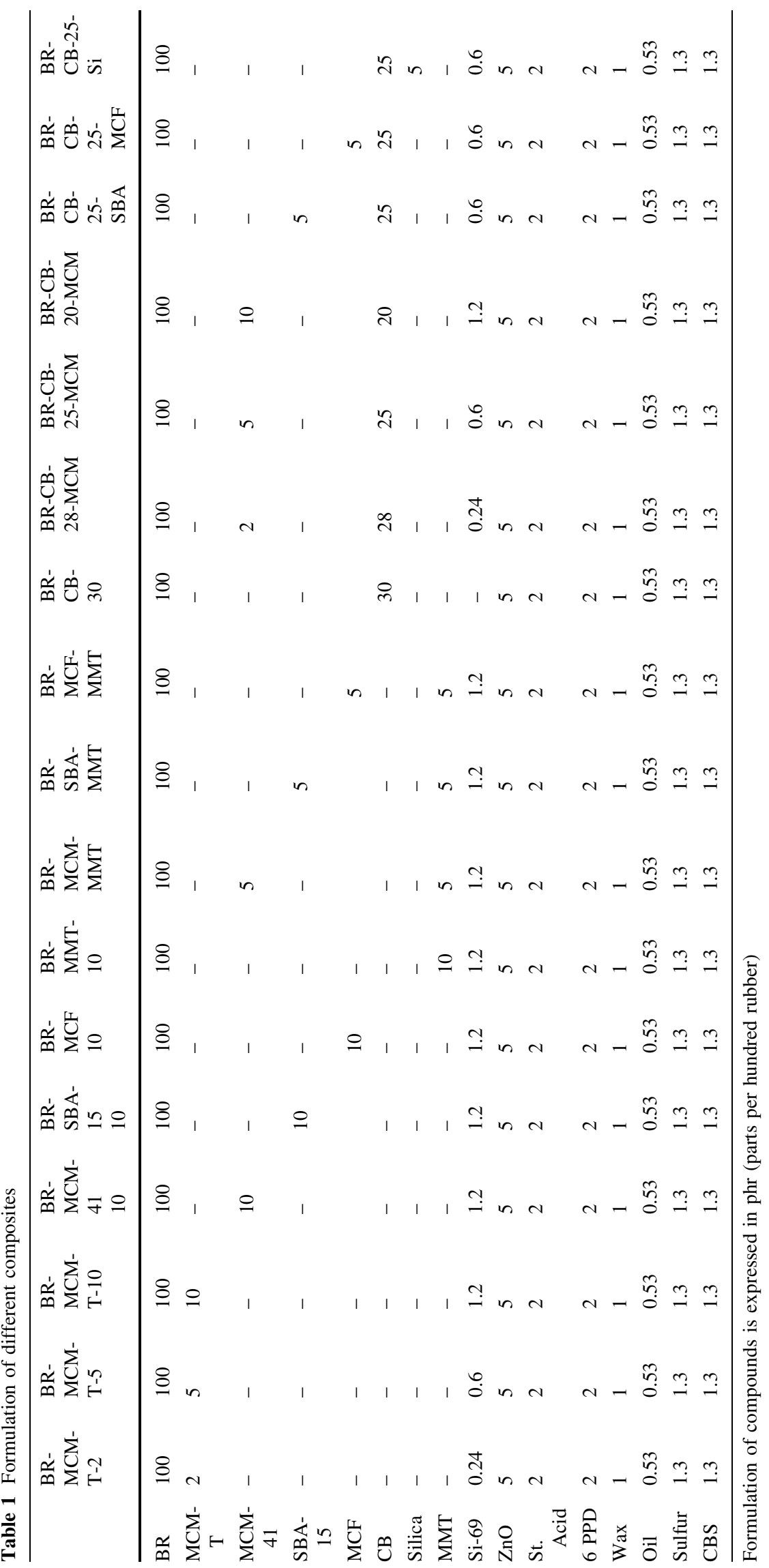




\section{Dynamic mechanical analysis (DMA)}

Dynamic mechanical analysis is done using Metravib (Model 450), France, in tension compression mode: frequency $-1 \mathrm{~Hz}$; strain-0.01\% and temperature ramp: -140 to $+80{ }^{\circ} \mathrm{C}$ at $2{ }^{\circ} \mathrm{C} / \mathrm{min}$. Test method used was in line with: DMA: ISO4664, ASTM D5992.

\section{Results and discussion}

\section{BET surface area analysis}

The BET surface area and pore volume of the various synthesized mesoporous silicates are given in Table 2. All MPS shows very high surface area. The pore size provides enough space for the polymer chains to enter into the pores [14].

\section{X-ray diffraction analysis (XRD)}

Figure 1a shows the XRD patterns of the MCM-41 (calcined) sample. MCM-41 shows a sharp XRD peak around $2 \theta=2^{\circ}$, less intense peaks around $2 \theta=3.8$ and $4.4^{\circ}$. This indicates the well-ordered hexagonal structure of the material is formed [20]. Similarly, Fig. 1b displays the XRD pattern of the SBA-15 (calcined) sample. Like MCM$41, \mathrm{SBA}-15$ also shows a strong peak around $2 \theta=1.5^{\circ}$ which corresponds to the (100) plane and showing a highly regular order. In the XRD pattern of MCF (Fig. 1c) no peak can be observed which confirms that MCF is an amorphous material as reported in the literature [20].

\section{Transmission electron microscopy (TEM) analysis}

Transmission electron micrographs of the mesoporous samples were shown in Fig. 2. The TEM images (Fig. 2a) confirm the regular hexagonal array of uniform channels for MCM-41 [21]. TEM micrographs (Fig. 2b) of SBA-15 sample substantiate the well-ordered, onedimensional pore structure of the material as reported in literature [22]. From these micrographs it was calculated that the average pore size of MCM-41 is around $3 \mathrm{~nm}$ and for SBA-15 and MCF it was around 4-6 nm, which corroborates well with the results obtained from BET analysis.
TEM micrographs in Fig. 2c do not show any longrange ordering. The structure is most probably similar to a disordered worm hole morphology of MCF [23].

\section{SEM analysis of mesoporous silica}

MCM-41 shows more regular shape, a well-defined spherical morphology with particle size range from 300 to 500 nm (Fig. 3a). From SEM images shown in Fig. 3b, it can be seen that SBA-15 has a typical wheat-like morphology and consists of aggregates of uniform rope-like particles. As can be seen in Fig. 3c, a homogeneous and highly porous structure was observed in the MCF material.

\section{Mechanical properties of $\mathrm{BR} / \mathrm{mesoporous} \mathrm{silica}$ (MPS) composites}

As the mesoporous silica are nanoparticles, hence the loading of these fillers was restricted to $\leq 10 \mathrm{phr}$. Table 3 reports the effect of filler loading of MCM-T (with template) on the mechanical properties of the composites.

The tensile strength, tensile modulus and hardness of the composite materials increase as the amount of filler is increased. It is remarkable that all the composite materials are having a high mechanical strength value compared to virgin matrix. The tensile strength of BR-MCM-T-10 is $65 \%$ higher in comparison to BR matrix.

Hence, $10 \mathrm{phr}$ loading was optimized for further studies. At this optimized loading, composites were made out of different Mesoporous Silica (MPSs). Table 4 reports the mechanical properties of composites made out of various MPSs.

A good interface between the MPSs and BR matrix is very important and required for a material to withstand the stress when subjected to an applied load. Figure 4 furnishes a clear idea on how the structure of the filler depends upon the mechanical properties of the composite. MCM-T is the precursor of MCM-41 having a structure where the mesopores are filled by the surfactant or template (Fig. 5a). After the calcination of MCM-T, the structure changes to a mesoporous framework where the pores are available for anchoring/entering of the polymer chains (Fig. 5b). Commonly a filler will just fills the space between two polymer chains. But, the distinguish property of MPSs is that it not only fills the space between the polymer chains, but also enhances the anchoring/entering of polymer chains inside
Table 2 BET analysis of different mesoporous silicates

\begin{tabular}{llll}
\hline Sample & BET surface area, $\mathrm{m}^{2} / \mathrm{g}$ & Pore volume, $\mathrm{cm}^{3} / \mathrm{g}$ & Pore size, \\
\hline MCM-41 & 297 & 0.20 & 27 \\
MCF & 396 & 0.37 & 38 \\
SBA-15 & 446 & 0.43 & 38 \\
\hline
\end{tabular}


Fig. 1 XRD of various synthesized MPS

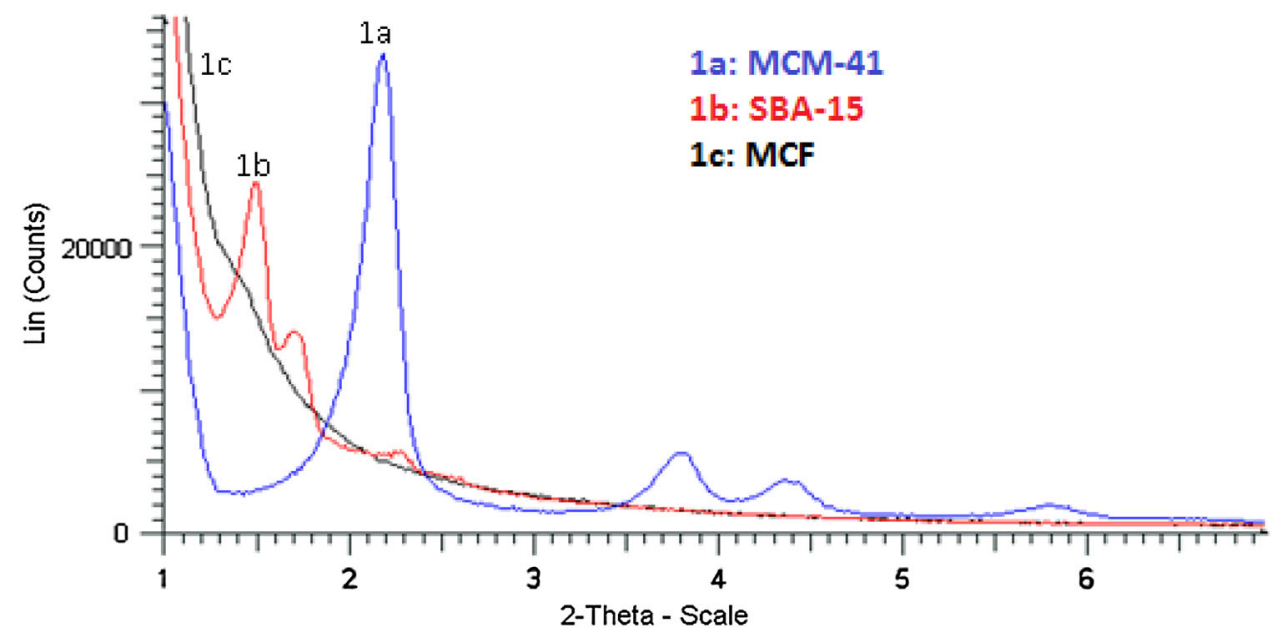

the pores which results in a substantial improvement in the tensile strength as shown in Fig. 4. BR-MCM-T shows little improvement as its pores are filled with template. However, it is quite remarkable that 10 phr BR-MCM-41loaded matrix increases tensile strength almost $250 \%$ as compared to virgin matrix. It implies that mesoporous material enhances the mechanical properties of the matrix up to a great extent.

The tensile strength and modulus of the composites using MCM-41 as filler were remarkably higher than that of MCF and SBA. It may be due to the peculiar properties of MCM-41 like, highly ordered periodic arrays of uniformly sized channels, narrow pore size distribution and long-range ordering. In the synthesis of polymer nanocomposite, agglomeration of particles or fillers is a major issue. Here also from the BET analysis, it is profoundly clear that SBA-15 is having a high surface area in comparison with MCM-41 which consequences the agglomeration of particles, end up with a drop in the tensile strength value. When the filler particles have very higher surface area, the filler-filler interaction increases and it would be higher than the polymer-filler interaction. At this point the particles will try to agglomerate and it will be difficult to be dispersed. The agglomerates of MCF fillers are visible in the SEM image of BR-MCF-10, which shows minimum property improvement among the three types of MPSs (Fig. 5c). The better dispersion of MCM-41 can be seen in Fig. 5 d.

\section{Mechanical properties of BR-MPSs-montmorillonite (MMT) hybrid composites}

With encouraging properties from the mesoporous composites, hybrid composites using a combination of fillers of different shape and morphology in the BR matrix were prepared. Table 5 reports the mechanical properties of BRMPS-MMT hybrid composites.

When two different shaped fillers are used in a matrix, it is very essential that both of them should have good interfacial adhesion with the matrix. The tensile values of the MPS-MMT composites illustrate that the interfacial interaction between the particles and the matrix diminishes due to very high surface area of both the nanofillers. Previously it is reported that MMT and MCM can be utilized as reinforcing filler in PP at lower filler loading [25]. But, the synergic effect that worked for PP is not happening in the case of BR due to the structural difference between them and also due to high filler loading. However, it is important to note that almost all the mechanical properties of BR-MCF-MMT are superior to BR-MCF-10 as well as BR-MMT-10, while the other two MPSs show a collapse in their properties, compared to the only presence of MPSs. This improvement in the case of MCF is owing to the stress transfer between the matrix and clay layers when a load is applied [26].

Figure 6a-c displays the compiled X-ray diffractogram image of MMT, BR-MCF-MMT and BR-MCF-MCM. Figure 6d shows the XRD image of BR-SBA-MMT. Virgin MMT shows a sharp peak around $2 \theta$ values of 3.5 and 7.3. From the XRD patterns of the composites a clear idea can be received about why the tensile strength value is getting increased for MCF-MMT composite, compared to bare MCF composite. In BR-MCF-MMT there is a shift in the XRD peak position which clarifies the increase in the tensile strength due to intercalation of the layers of MMT. But, this trend is not observed in MCMMMT and SBA-MMT composites. In addition, the interplanar spacing values of MCF-MMT are higher compared to other MPSs which further explain the intercalation of MMT layers. 
Fig. 2 a TEM images of MCM-41 (at both low and high resolution). $\mathbf{b}$ TEM images of SBA-15 (at both low and high resolution). c TEM images of MCF (at both low and high resolution) a
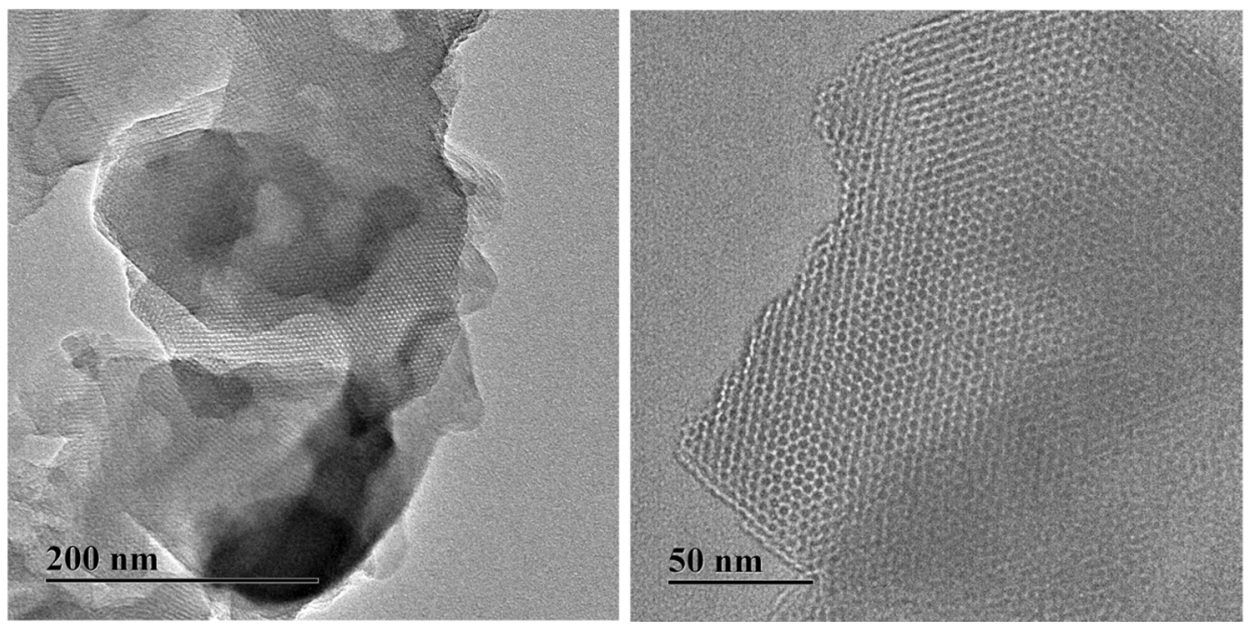

b
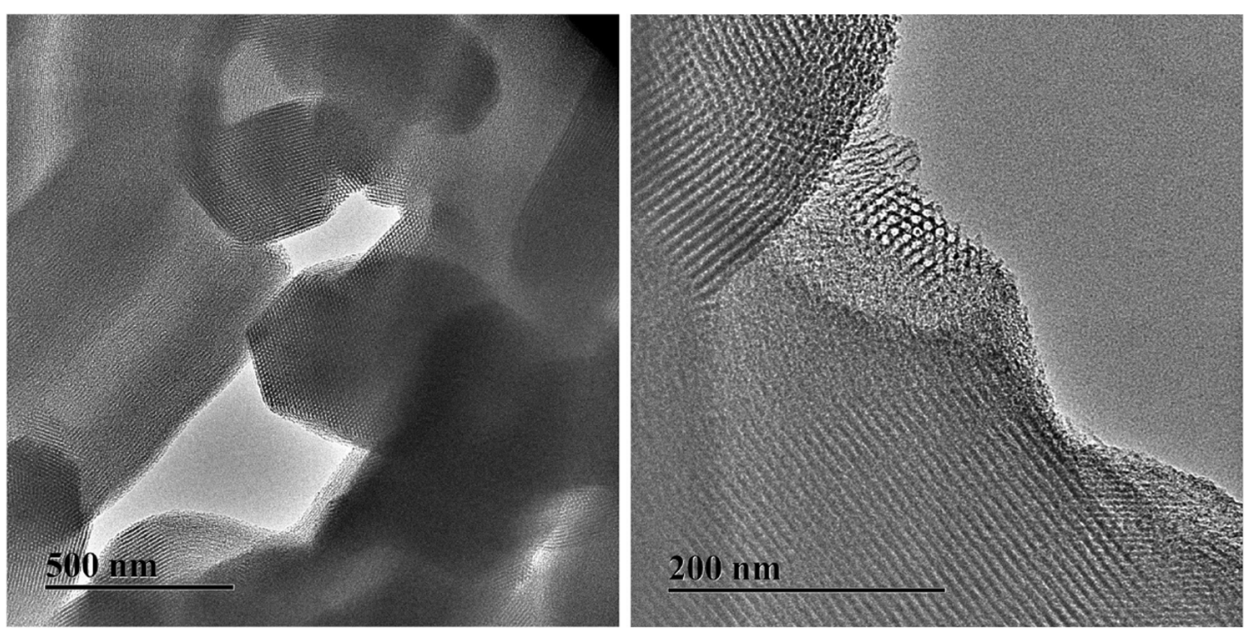

C

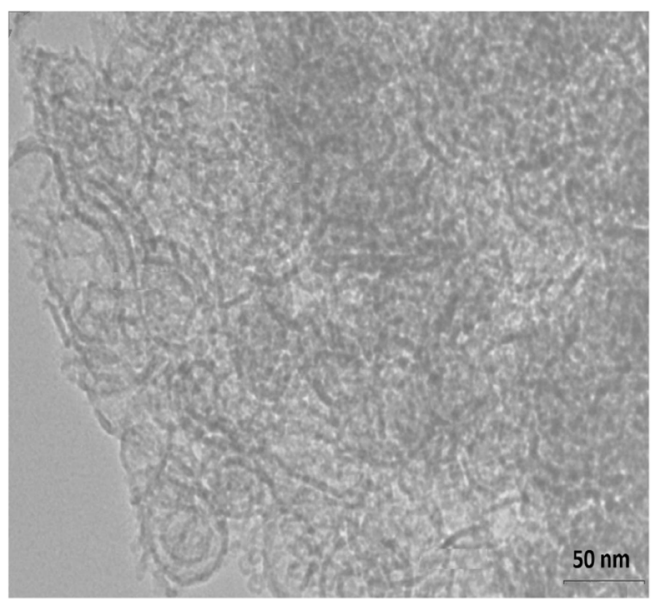

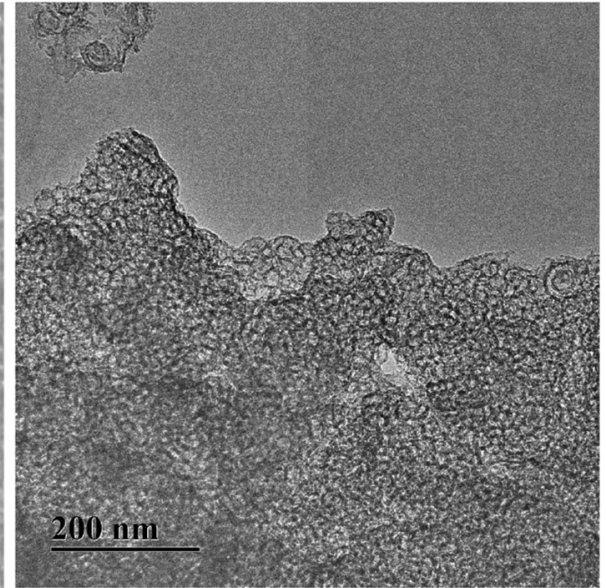

The dispersion of MCF is better in presence of MMT, as seen from the SEM of the composite (Fig. 6e), whereas the SBA fillers are not so homogeneously dispersed, agglomerated white particles can be seen in the SEM image (Fig. 6f).

\section{Mechanical properties of carbon black- MCM-41 hybrid composites}

The mechanical properties of the MCM and carbon blackbased hybrid composites are reported in Table 6 . 

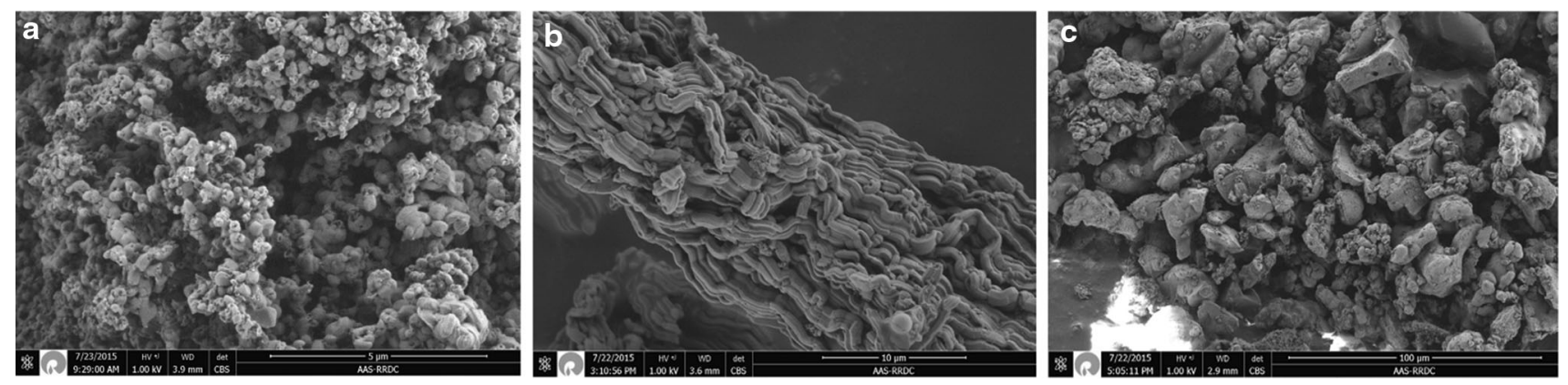

Fig. 3 SEM images of a MCM-41, b SBA-15 and c MCF

Table 3 Mechanical properties of the MCM-T/BR composite

\begin{tabular}{lcccc}
\hline & BR & BR-MCM-T-2 & BR-MCM-T-5 & BR-MCM-T-10 \\
\hline Tensile strength (MPa) & $1.19 \pm 0.09$ & $1.46 \pm 0.11$ & $1.57 \pm 0.11$ & $1.97 \pm 0.13$ \\
Elongation at break (\%) & $120 \pm 8$ & $134 \pm 7$ & $167 \pm 15$ & $161 \pm 14$ \\
$100 \%$ modulus (MPa) & $1.10 \pm 0.07$ & $1.40 \pm 0.10$ & $1.39 \pm 0.09$ & $1.70 \pm 0.12$ \\
Hardness (Shore A) & $42 \pm 1$ & $44 \pm 1$ & $45 \pm 1$ & $46 \pm 1$ \\
\hline
\end{tabular}

Table 4 Mechanical properties of calcined MPSs BR composite

\begin{tabular}{lcccc}
\hline & BR-MCM-T-10 & BR-MCM-41-10 & BR-SBA-15-10 & BR-MCF-10 \\
\hline Tensile strength (MPa) & $1.97 \pm 0.12$ & $4.07 \pm 0.22$ & $2.47 \pm 0.15$ & $2.27 \pm 0.14$ \\
Elongation at break (\%) & $161 \pm 12$ & $279 \pm 15$ & $325 \pm 12$ & $437 \pm 17$ \\
$100 \%$ modulus (MPa) & $1.70 \pm 0.07$ & $1.71 \pm 0.07$ & $2.3 \pm 0.10$ & $1.02 \pm 0.05$ \\
Hardness (Shore A) & $46 \pm 1$ & $43 \pm 1$ & $47 \pm 1$ & $45 \pm 1$ \\
\hline
\end{tabular}

Fig. 4 Optimization of MCM filler in BR matrix

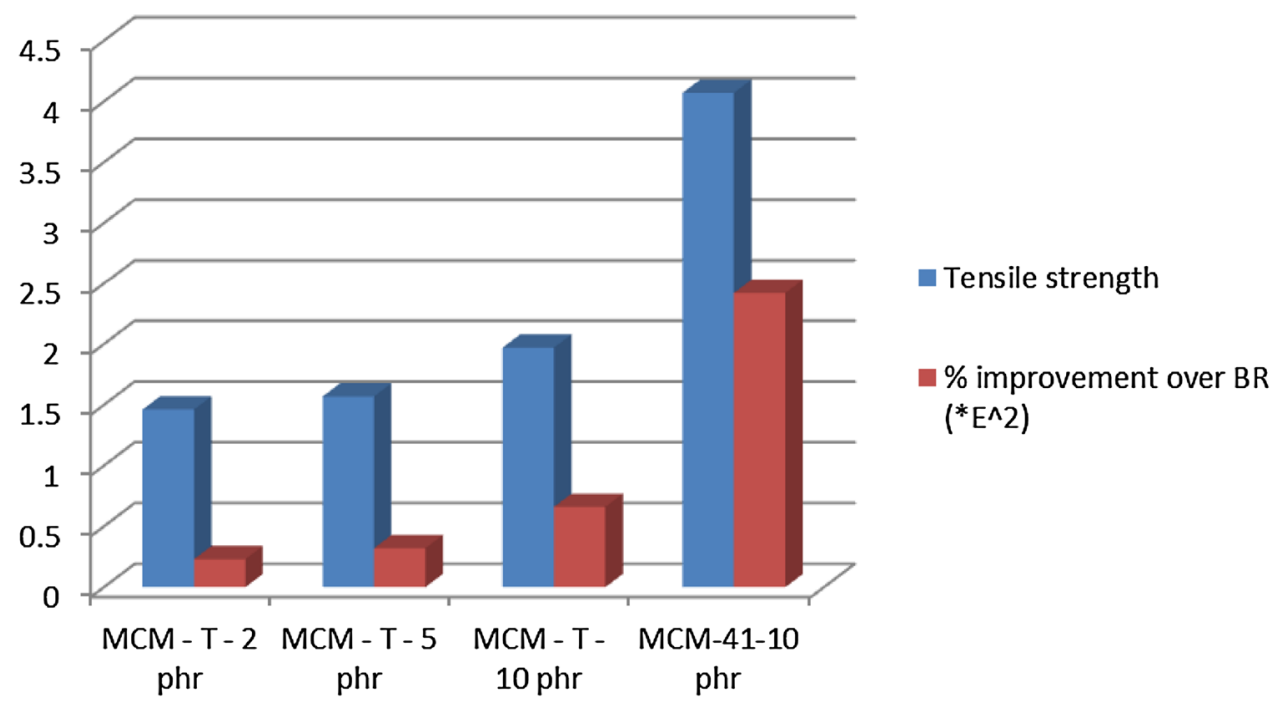

From the above table, it is evident that there is a small decrease in the tensile strength value of BR-CB-28 in comparison with BR-CB-30. When MCM-41 is added to the matrix, the expectation was the polymer molecule will anchor inside the pores of the MPS, which result in additional increase in the mechanical properties. At very low
MPS loading, e.g., $2 \mathrm{phr}$, enough numbers of polymer chains are not anchoring into the mesopores which effects in the drop of tensile strength. After this, the mechanical properties are getting increased in BR-CB-25 due to the anchoring of polymer inside the nano-sized pores of MPSs extending along the channels to the openings. It will not 
Fig. 5 Images of a MCM-T and b MCM-41 [24]. SEM image of cryo-fractured of c BR-MCF-10 and d BR-MCM41-10
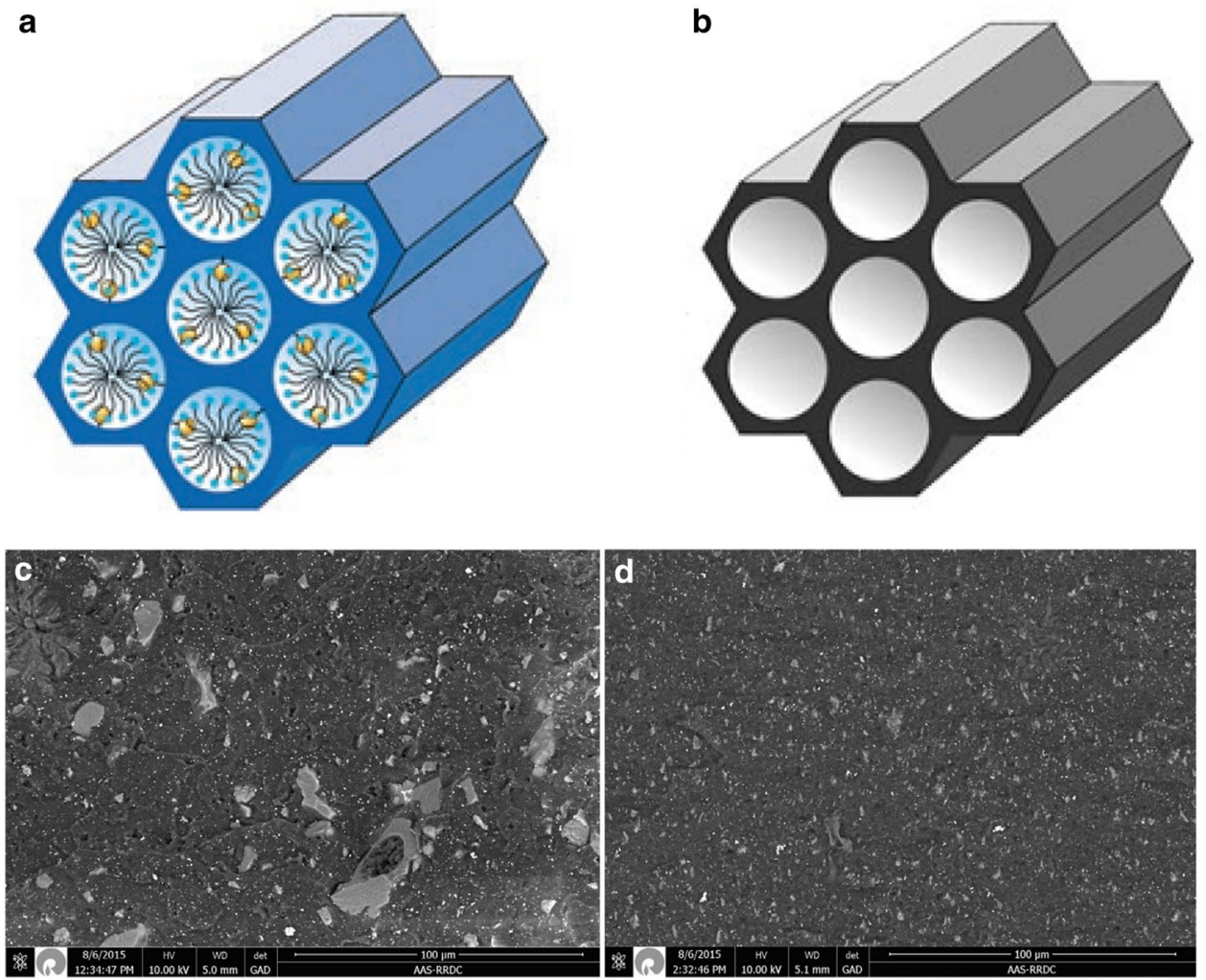

Table 5 Mechanical properties of various MPS-MMT composites

\begin{tabular}{llllc}
\hline & BR-MCM-41-MMT & BR-SBA-15-MMT & BR-MCF-MMT & BR-MMT-10 \\
\hline Tensile strength (MPa) & $2.89 \pm 0.13$ & $2.64 \pm 0.13$ & $3.08 \pm 0.17$ & $2.00 \pm 0.13$ \\
Elongation at break (\%) & $463 \pm 30$ & $291 \pm 15$ & $352 \pm 20$ & $524 \pm 30$ \\
$300 \%$ modulus (MPa) & $2.04 \pm 0.04$ & - & $2.65 \pm 0.12$ & $0.95 \pm 0.08$ \\
Hardness (Shore A) & $48.0 \pm 1$ & $48.5 \pm 1$ & $50.5 \pm 1$ & $40 \pm 1$ \\
\hline
\end{tabular}

- means that the value is empty in this case, as the maximum elongation is less than $300 \%$ only enhance the miscibility through the entanglement and inter-diffusion between the matrix and the particulate, but also highly suppress the aggregation of fillers. At higher loading of MPSs such as $10 \mathrm{phr}$, in BR-CB-20, the nanofillers may agglomerate leading to drop in properties.

\section{Comparison of BR-CB-25-MCM with other MPS and silica}

Figure 7 provides a comparison of BR-CB-25 with various MPS and precipitated silica. It is emphasizing from the graph that mesoporous silica, MCM-41 is enhancing the BR matrix almost double the value compared to the precipitated silica. Here the polymer is getting introduced into the mesopores of MPS which enhances the distribution/ dispersion of the filler in the matrix which in turn boost the mechanical properties. Out of various MPSs, MCM is producing the best results compared to MCF and SBA.

\section{Dynamic mechanical analysis (DMA)}

The enhancement of mechanical properties can be further evaluated by DMA. The storage modulus and loss factor $(\tan \delta)$ versus temperature are plotted in Fig. 8.

It is quite evident from Fig. 8a that the $\tan \delta$ peak height (mechanical damping efficiency) reduces in all the composites along with $10 \mathrm{phr}$ MCM-41-loaded BR composite as compared to virgin BR matrix conversely, the peak broadens slightly for hybrid composites. It can also be seen from Fig. 8a that the $\mathrm{T}_{g}$ of all the hybrid composites has been shifted slightly towards right. The reduction of $\tan \delta$ height and shifting of $\mathrm{T}_{g}$ towards higher temperature can be attributed to the restricted mobility of the polymer chains due to better polymer-filler interaction. Similarly, it is also observed that, with incorporation of filler, the storage modulus increases which accounts for the reinforcing action of filler present in the BR matrix (Fig. 8b). This indicates that 
Fig. 6 XRD images of a MMT, b BR-MCM-MMT and c BRMCF-MMT. d XRD image of BR-SBA-MMT. SEM images of e BR-MCF-MMT and $\mathbf{f}$ BRSBA-MMT
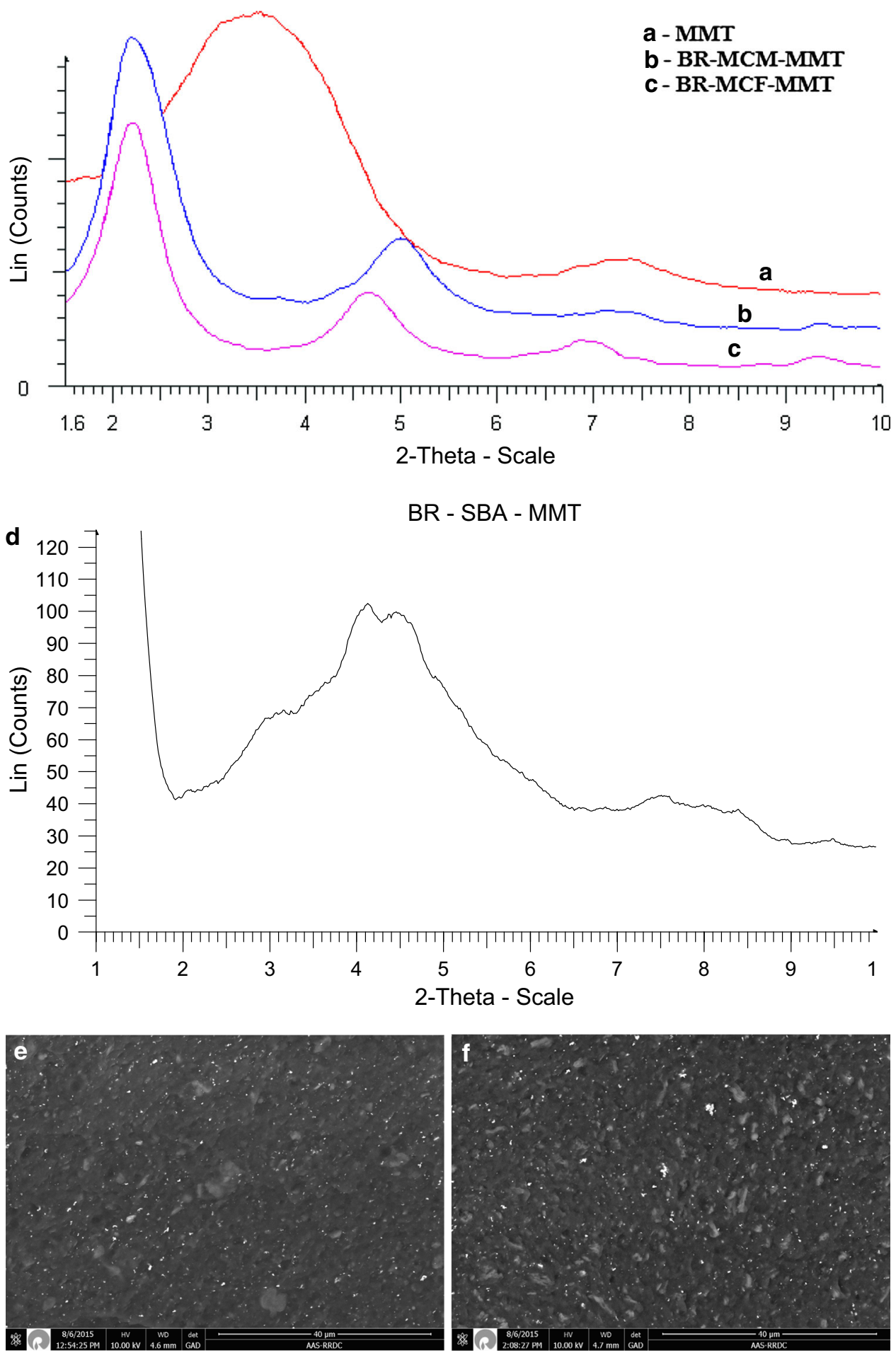

Table 6 Mechanical properties of MCM and carbon black hybrid composites

\begin{tabular}{lcccc}
\hline & BR-CB-30 & BR-CB-28-MCM & BR-CB-25-MCM & BR-CB-20-MCM \\
\hline Tensile strength (MPa) & $6.46 \pm 0.28$ & $5.94 \pm 0.30$ & $7.76 \pm 0.41$ & $5.80 \pm 0.35$ \\
Elongation at break (\%) & $522 \pm 20$ & $397 \pm 12$ & $556 \pm 22$ & $560 \pm 21$ \\
$300 \%$ modulus (MPa) & $2.86 \pm 0.17$ & $3.61 \pm 0.19$ & $3.42 \pm 0.17$ & $2.70 \pm 0.12$ \\
Hardness (Shore A) & $54 \pm 1$ & $54 \pm 1$ & $54 \pm 1$ & $43 \pm 1$ \\
\hline
\end{tabular}


Fig. 7 Comparison of tensile strength of MCM-based hybrid composite with other MPS and silica-based ones

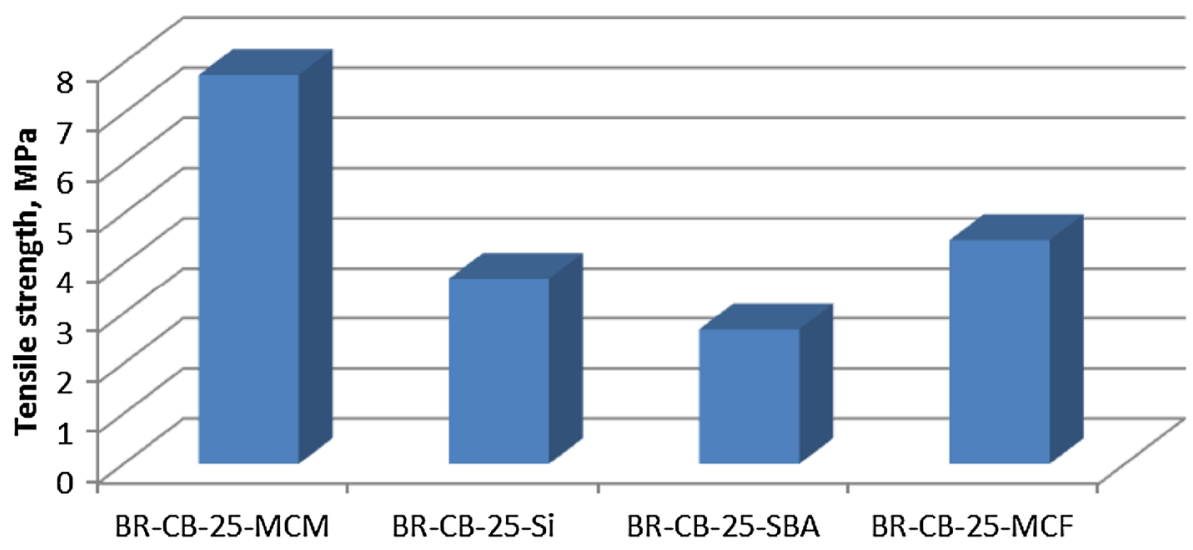

strength. These results are in good agreement with the aforementioned mechanical property results.

\section{Conclusions}

Different mesoporous silicates were synthesized and subsequently incorporated into polybutadiene rubber (BR) in presence of silane coupling agents. Mechanical properties of all the composites were significantly improved as compared to the pure BR matrix. 10 phr BR-MCM-41loaded matrix increased tensile strength almost $250 \%$ in comparison to virgin matrix. The hybrid composites were synthesized by a co-incorporation of two different kinds of fillers into the BR matrix, and gained a better mechanical properties in the case of BR-MCF-MMT composite due to intercalation of MMT layers. Carbon black and MCM-41 were successfully co-incorporated in BR matrix and found that at $5 \mathrm{phr}$ MCM-41 and $25 \mathrm{phr}$ CB loading, the hybrid composite had maximum mechanical strength. Comparison of this tensile value with other mesoporous silicates and precipitated silica substantiated that MCM-41 enhanced the BR matrix almost double the value compared to the precipitated silica. This is owing to the fact that the polymer is getting introduced into the mesopores of MCM-41 which enhances the miscibility between the filler and the matrix which in turn boost the mechanical properties.

Acknowledgments The authors are highly thankful to Mr. Padmanabhan R. (National Institute of Technology, Calicut), Mr. Chirag S. Shah (RTG-VMD), Mr. S. Chatterjee (PAC-VMD), Mrs. Rashmi Dave (Analytical Lab., RTG-VMD) for their kind cooperation. The authors wish to thank the management of Reliance Industries Limited for giving consent to publish the work.

Open Access This article is distributed under the terms of the Creative Commons Attribution 4.0 International License (http://creative commons.org/licenses/by/4.0/), which permits unrestricted use, distribution, and reproduction in any medium, provided you give appropriate credit to the original author(s) and the source, provide a link to the Creative Commons license, and indicate if changes were made. 


\section{References}

1. Guido KB (2003) Concepts for the incorporation of inorganic building blocks into organic polymers on a nanoscale. Prog Polym Sci 28:83-114

2. Pan LJ, He PS, Zou G, Chen DZ (2003) PbS/epoxy resin nanocomposite prepared by a novel method. Mater Lett 58:176-178

3. Sui G, Zhong WH, Yang XP, Yu YH (2008) Curing kinetics and mechanical properties of carbon nanotube/natural rubber composites. Mater Sci Eng A 485:524-531

4. Nakason C, Kaesaman A, Eardrod K (2007) Cure and mechanical properties of natural rubber- $<\mathrm{i}>\mathrm{g}</ \mathrm{i}>$-poly (methyl methacrylate)-cassava starch compounds. Mater Lett 59:4020-4025

5. Wang N, Fang Q, Zhang J, Chena E, Zhang X (2011) Incorporation of nano-sized mesoporous MCM-41 material used as fillers in natural rubber composite Mater Sci Eng A 528:3321-3325

6. Behrens P (1993) Mesoporous inorganic solids. Adv Mater 5:127-132

7. Zhao XS, Lu GQ, Millar GJ (1996) Advances in mesoporous molecular sieve MCM-41. Ind Eng Chem Res 35:2075-2090

8. Zhang FA, Song C, Yu CL (2011) Effects of preparation methods on the property of PMMA/SBA-15 mesoporous silica composites. J Polym Res 18:1757-1764

9. Yang J, Huang L, Zhang Y, Chen F, Zhong M (2013) Mesoporous silica particles grafted with polystyrene brushes as a nucleation agent for polystyrene supercritical carbon dioxide foaming. J Appl Polym Sci 130:4308-4317

10. Shen BH, Hsieh ML, Chen HY, Wu JY (2013) The preparation of hollow silica spheres with mesoporous shell via polystyrene emulsion latex template and the investigation of ascorbic acid release behavior. J Polym Res 20:220-231

11. Nur H, Rahman NA, Endud S, Wei LK (2007) Thermal stability of conductivity of composite comprising polyaniline and MCM41 Malaysian. Polym J 2:12-21

12. Chen Z, Song C, Bai R, Wei Z, Zhang F (2012) Effects of mesoporous SBA-15 contents on the properties of polystyrene composites via in situ emulsion polymerization. J Polym Res 19:9846-9853

13. Moussaif N, Crespo C, Meier JG, Jimenez MA (2012) Synergistic reinforcement of nanoclay and mesoporous silicate fillers in polycaprolactone: the effect of nanoclay on the compatibility of the components. Polymer 53:3741-3748
14. Wei L, Hu N, Zhang Y (2010) Synthesis of polymer-mesoporous silica nanocomposites. Materials 3:4066-4079

15. Pérez LD, Giraldo LF, López BL, Hess M (2006) Reinforcing of elastomers with mesoporous silica. Macromol Symp 245:628-640

16. López BL, Pérez LD, Mesa M, Sierra L, Devaux E, Camargo M, Campagne C, Giraud S (2005) Use of mesoporous silica as a reinforcing agent in rubber compounds. E-Polymer 018:1-13

17. Luan Z, Cheng CF, Zhou W, Klinowski J (1995) Mesopore molecular sieve MCM-41 containing framework aluminum. J Phys Chem 99:1018-1024

18. Zhao D, Huo Q, Feng J, Chmelka BF, Stuck GD (1998) Nonionic triblock and star diblock copolymer and oligomeric surfactant syntheses of highly ordered, hydrothermally stable, mesoporous silica structures. J Am Chem Soc 120:6024-6028

19. Lettow JS, Han Y, Schmidt-Winkel JP, Yang PD, Zhao DY, Stucky GD, Wing JY (2000) Hexagonal to mesocellular foam phase transition in polymer-templated mesoporous silicas. Langmuir 16:8291-8297

20. Sayari A, Liu P, Kruk M, Jaroniec M (1997) Characterization of large pore MCM-41 molecular sieves obtained via hydrothermal restructuring. Chem Mater 9:2499-2506

21. Jeong SY, Suh JK, Lee MJ, Kwon OY (1997) Preparation of Silica-based mesoporous materials from fluorosilicon compounds: gelation of $\mathrm{H} 2 \mathrm{SiF} 6 \mathrm{in}$ ammonia surfactant solution. J Colloid Interface Sci 192:156-162

22. Wang X, Lin KSK, Chan JCC, Cheng S (2005) Direct synthesis and catalytic applications of ordered large pore aminopropylfunctionalized SBA-15 mesoporous materials. J Phys Chem B 109:1763-1769

23. Pauly TR, Liu Y, Pinnavia TJ, Billinge SJL, Ricker TP (1999) Textural mesoporosity and the catalytic activity of mesoporous molecular sieves with wormhole framework structures. J Am Chem Soc 121:8835-8841

24. Hoffmann F, Cornelius M, Morell J, Michael F (2006) Angew Chem Int Ed 45:3216-3251

25. Wang N, Zhaoa C, Shia Z, Shaob Y, Lia H, Gao N (2009) Coincorporation of MMT and MCM-41 nanomaterials used as fillers in PP composite. Mater Sci Eng B 157:44-47

26. Wang N, Fang Q, Zhang J, Chena E, Zhang X (2011) Incorporation of nano-sized mesoporous MCM-41 material used as fillers in natural rubber composite. Mater Sci Eng A 528:3321-3325 